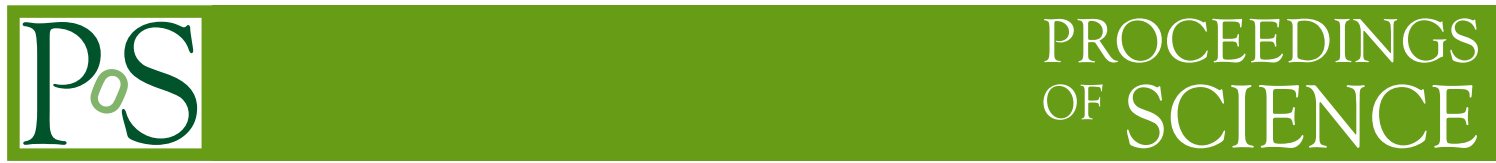

\title{
Chiral two-nucleon dynamics, analyticity and dispersion relations
}

\author{
A. M. Gasparyan* \\ Institut für Theoretische Physik II, Fakultät für Physik und Astronomie, Ruhr-Universität \\ Bochum, 44780 Bochum, Germany; \\ SSC RF ITEP, Bolshaya Cheremushkinskaya 25, 117218 Moscow, Russia \\ E-mail: ashot.gasparyan@rub.de

\section{E. Epelbaum} \\ Institut für Theoretische Physik II, Fakultät für Physik und Astronomie, Ruhr-Universität \\ Bochum, 44780 Bochum, Germany
}

\section{M.F.M. Lutz}

GSI Helmholtzzentrum für Schwerionenforschung GmbH,

Planckstraße 1, 64291 Darmstadt, Germany

\begin{abstract}
Nucleon-nucleon interaction is studied using relativistic form of the chiral Lagrangian. Partialwave amplitudes are computed in chiral perturbation theory at next-to-next-to-leading order in the subthreshold region. The most general constraints set by analyticity and unitarity are implemented to extrapolate the amplitudes into the physical region. Empirical phase shifts are described up to laboratory energies $T_{\mathrm{lab}} \simeq 250 \mathrm{MeV}$. The issues related with renormalization and convergence of perturbation theory are investigated.
\end{abstract}

The 8th International Workshop on Chiral Dynamics, CD2015 ***

29 June 2015 - 03 July 2015

Pisa,Italy

\footnotetext{
${ }^{*}$ Speaker.
} 


\section{Introduction}

In recent years there have been many studies related to the application of the methods of effective field theory to the nucleon-nucleon interaction. The nucleon-nucleon system is quite different from the pure pionic sector or even the one-nucleon sector where the framework of chiral perturbation theory (ChPT) have been successfully applied (see e.g. [1]). In particular, a purely perturbative treatment in the physical near-threshold region of the nucleon-nucleon scattering is impossible, as follows from the existence of the deuteron bound state. Formally one can try to argue in favour of the non-perturbative nature of the nucleon-nucleon interaction by considering naive dimensional power counting for Feynman diagrams, and organizing the expansion in terms of the small parameter $Q$ given roughly by the ratio of the soft scale (pion mass or the nucleon momentum) and the hard scale (nucleon mass or the $\rho$-meson mass) starting with leading order $Q^{0}$, next-to-leading order $Q^{1}$, etc. The leading contribution of order $Q^{0}$ to the nucleon-nucleon scattering amplitude are generated by the leading contact interaction and the one-pion-exchange graph (see Fig.1 (a), (b)). The loop diagrams that are 2-nucleon irreducible start to contribute at order $Q^{2}$ (Fig.1 (f), (g), (h)). Nucleon-nucleon subleading contact interactions appear at the same order (Fig.1 (i)). On the other hand the 2-nucleon reducible 1-loop diagrams (Fig.1 (c), (d), (e)) are enhanced due to the so-called pinch singularity originating from the integration region where both intermediate nucleons are on-shell [2]. An analogous enhancement takes place for multiloop 2-nucleon reducible diagrams. Nevertheless the perturbative series could still converge as follows from the power counting arguments since there are always a finite number of diagrams contributing at each order. However this does not happen numerically in the real world for low partial waves as was already mentioned above. For higher partial waves the perturbative expansion seems to work reasonably well [3].

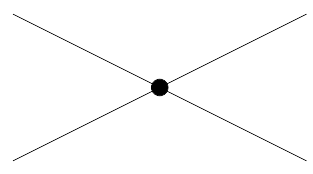

(a)

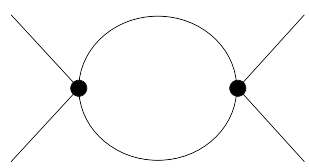

(c)

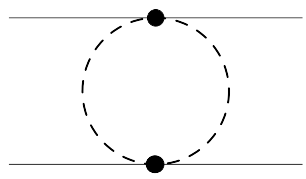

(f)

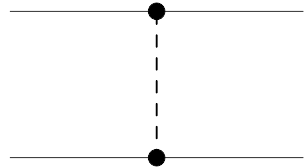

(b)

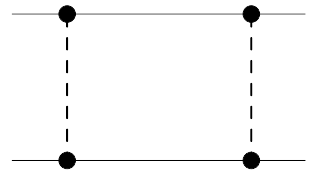

(d)

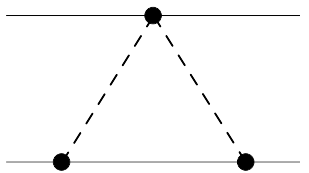

(g)

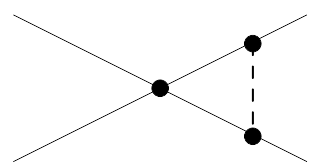

(e)

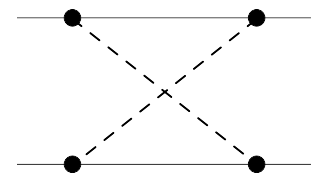

(h)

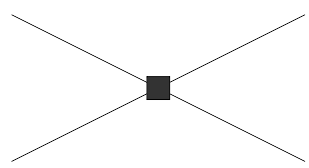

(i)

Figure 1: Tree and one-loop diagrams contributing to the nucleon-nucleon scattering amplitude at chiral orders $Q^{0}((\mathrm{a}),(\mathrm{b})), Q^{1}((\mathrm{c}),(\mathrm{d}),(\mathrm{e})), Q^{2}$ ((f), (g), (h), (i)) according to naive dimensional power counting.

There are several schemes that implement the non-perturbative dynamics of the nucleon- 
nucleon interaction based on the chiral Lagrangian. The most popular scheme originally proposed by Weinberg [2] is the potential approach. In this scheme one resums all 2-nucleon reducible diagrams by solving the Lippmann-Schwinger-type equation and applies a power counting to the potential that consists of all possible 2-nucleon irreducible contributions (see e.g. [4, 5]). In a different approach of Kaplan, Savage and Wise [6] only the leading order contact interaction is resummed whereas pions are treated perturbatively. This scheme, however, was shown not to converge for some of the channels [7].

The basic principle of effective field theory is to calculate the most general $S$-matrix consistent with analyticity, unitarity and symmetries of the underlying theory by means of the most general effective Lagrangian [8]. In the case of a strict ChPT unitarity is satisfied only perturbatively. In the case of the non-perturbative nucleon-nucleon interaction it is not obvious that already mentioned standard methods obey this basic principle. In order to understand this issue better we start directly with the principles of analyticity and unitarity using dispersion relations but, nevertheless, performing a matching to the ChPT Lagrangian using techniques developed and successfully applied in Refs. $[9,10,11,12]$.

\section{Making use of analyticity and unitarity}

Analyticity and s-channel unitarity (relevant in the physical threshold region) allow one to write down the discontinuity of a partial wave amplitude along the right-hand cut (neglecting inelastic channels) in the form

$$
\frac{1}{2 i}(T(s+i \varepsilon)-T(s-i \varepsilon))=T(s+i \varepsilon) \rho^{(J P)}(s) T(s-i \varepsilon),
$$

where $T(s)$ and $\rho(s)$ are the scattering amplitude and the phase space factor respectively (they turn into $2 \times 2$ matrices for the coupled partial waves). This equation can be rewritten in the integral form as a partial wave dispersion relation with one subtraction at $s=\mu_{M}^{2}$

$$
T(s)=U(s)+\int_{4 m_{N}^{2}}^{\infty} \frac{d s^{\prime}}{\pi} \frac{s-\mu_{M}^{2}}{s^{\prime}-\mu_{M}^{2}} \frac{T(s) \rho\left(s^{\prime}\right) T^{*}\left(s^{\prime}\right)}{s^{\prime}-s-i \varepsilon} .
$$

Here $U(s)$ is the generalized potential, i.e. the part of the amplitude containing only the left-hand singularities. If the quantity $U(s)$ is known along the whole right-hand cut the equation (2.2) can be solved by means of the $N / D$ method [13]. The scattering amplitude $T(s)$ is represented as

$$
T(s)=D^{-1}(s) N(s),
$$

where $D(s)$ has no singularities but the right-hand $s$-channel unitarity cut whereas the branch points of $N(s)$ correspond to those of $U(s)$. The non-linear equation (2.2) reduces to a system of linear equations for $N(s)$ and $D(s)$

$$
\begin{aligned}
& N(s)=U(s)+\int_{4 m_{N}^{2}}^{\infty} \frac{d s^{\prime}}{\pi} \frac{s-\mu_{M}^{2}}{s^{\prime}-\mu_{M}^{2}} \frac{N\left(s^{\prime}\right) \rho\left(s^{\prime}\right)\left[U\left(s^{\prime}\right)-U(s)\right]}{s^{\prime}-s}, \\
& D(s)=1-\int_{4 m_{N}^{2}}^{\infty} \frac{d s^{\prime}}{\pi} \frac{s-\mu_{M}^{2}}{s^{\prime}-\mu_{M}^{2}} \frac{N\left(s^{\prime}\right) \rho\left(s^{\prime}\right)}{s^{\prime}-s} .
\end{aligned}
$$

Note that an alternative technique based on the integration along the left-hand cut was utilized in Refs. [14, 15, 16, 17]. 


\section{Matching to the chiral Lagrangian}

A key ingredient of our work that allows us to draw a connection to the chiral effective Lagrangian is an assumption that the perturbative expansion of the nucleon-nucleon amplitude (discussed in Introduction) converges in the subthreshold region far away from the $s$-channel unitarity cut and from the part of the left-hand $t$-channel cut that corresponds to high momenta of the exchanged pions. The reasonableness of this assumption was nicely illustrated by the example of a toy model in Ref. [18]. The validity of perturbative expansion in the subthreshold region enables us to chose the subtraction point $\mu_{M}^{2}$ in Eq. (2.2) sufficiently below threshold (our typical choice is $\mu_{M}^{2}=4 m_{N}^{2}-2 M_{\pi}^{2}$ ) where the amplitude can be calculated from chiral Lagrangian using ChPT and can be matched to the non-perturbative solution of Eq. (2.2).

In the system of equations (2.4) one needs to know the function $U(s)$ along the whole righthand cut. In order to reliably extrapolate $U(s)$ into the physical region we split $U(s)$ into two parts

$$
U(s)=U_{\text {inside }}(s)+U_{\text {outside }}(s)
$$

where $U_{\text {inside }}(s)$ (in the notation of Ref. [9]) contains the closest part of the $t$-channel cut

$$
U_{\text {inside }}(s)=\int_{\Lambda^{2}}^{4 m_{N}^{2}-M_{\pi}^{2}} \frac{d s^{\prime}}{\pi} \frac{\Delta T\left(s^{\prime}\right)}{s^{\prime}-s} .
$$

The discontinuity $\Delta T(s)$ in this region is also calculated in chiral perturbation theory and the lower limit of the integral is chosen to be $\Lambda^{2}=4 m_{N}^{2}-9 M_{\pi}^{2}$ (the three-pion-exchange threshold). Note that we use a covariant (relativistic) version of the ChPT for the nucleon-nucleon amplitude (see e.g. $[19,20])$ in order not to destroy analytic properties of the amplitudes in the vicinity of the $t$-channel cut.

The remaining part of $U(s), U_{\text {outside }}(s)$, is a smooth function of energy and can be analytically continued into the physical region by means of the conformal mapping technique [12]. Because of the matching condition at $s=\mu_{M}^{2}$ the coefficients of the conformal expansion are in one-toone correspondence with the low energy constants of the effective Lagrangian, which are free parameters to be fitted to data.

\section{Results}

The free parameters in our scheme were determined from the fit to the Nijmegen partial wave analysis (PWA) [21]. Another PWA [22] is used for comparison. For the fit the energy points below $T_{\text {lab }}=100 \mathrm{MeV}$ are taken into account in order to correctly reproduce the threshold physics. The results of our fit at chiral orders $Q^{0}-Q^{3}$ are shown in Fig. 2 (Fig. 3) for the lowest uncoupled (coupled) partial waves. The quality of the fit is comparable with the one obtained in the potential approach. In particular, the convergence pattern from the order $Q^{0}$ to $Q^{3}$ looks very reasonable. Another indication of the convergence of the perturbative expansion in the subthreshold region are the values of the amplitude at the matching point $s=\mu_{M}^{2}$ after subtracting the dominant one-pionexchange contribution. They are listed in Table 1 (Table 2) for the uncoupled (coupled) partial waves. 

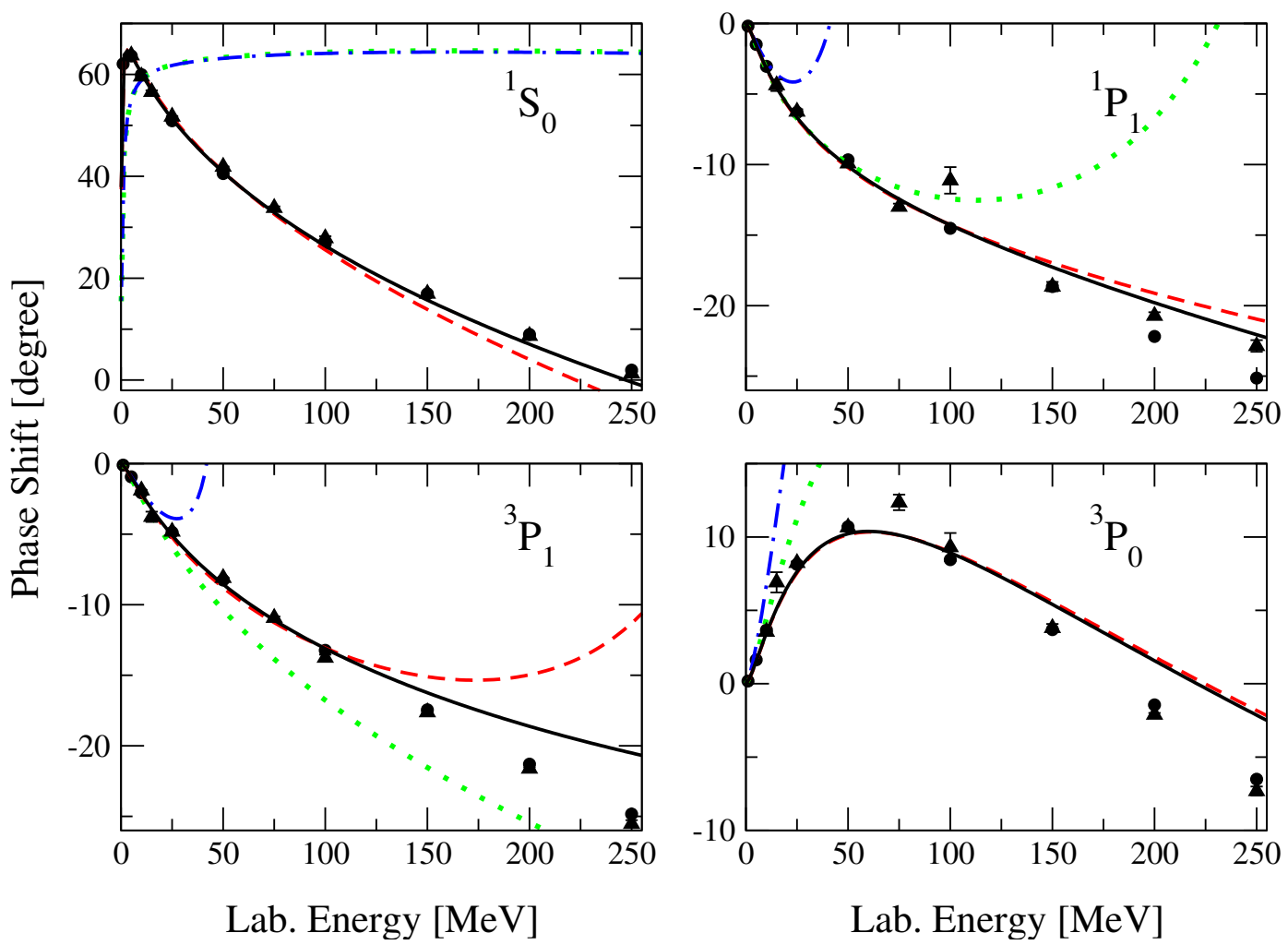

Figure 2: Neutron-proton phase shifts in uncoupled S- and P-waves calculated at orders $Q^{0}$ (dotted lines), $Q^{1}$ (dash-dotted lines), $Q^{2}$ (dashed lines) and $Q^{3}$ (solid lines) in comparison with the Nijmegen [21] (filled circles) and SAID [23, 22] (filled triangles) PWAs.

\begin{tabular}{|l|c|c|c|c|}
\hline & ${ }^{1} S_{0}$ & ${ }^{1} P_{1}$ & ${ }^{3} P_{1}$ & ${ }^{3} P_{0}$ \\
\hline$Q^{0}$ & $5.79 \times 10^{2}$ & & & \\
\hline$Q^{1}$ & $5.82 \times 10^{2}$ & & & \\
\hline$Q^{2}$ & $8.53 \times 10^{2}$ & $1.42 \times 10^{3}$ & $3.46 \times 10^{3}$ & $-6.43 \times 10^{3}$ \\
\hline$Q^{3}$ & $8.63 \times 10^{2}$ & $2.19 \times 10^{3}$ & $4.81 \times 10^{3}$ & $-5.70 \times 10^{3}$ \\
\hline
\end{tabular}

Table 1: Generalized potential at the matching point $U\left(\mu_{M}^{2}\right)$ at different chiral orders after subtracting onepion exchange contribution. Uncoupled partial waves.

Finally we addressed a question of the uniqueness of the solutions obtained by our method since the principles of effective field theory tell us that we have to find the most general $S$-matrix. It is known that the non-linear equation (2.2) may possess multiple solutions due to the so-called Castillejo-Dalitz-Dyson poles [24, 25]. Another way to see it is to modify the short range part of the generalized potential $U(s)$ by varying the cutoff $\Lambda$. In Fig. 4 we show the predictions (there are no free parameters in $D$-waves at order $Q^{3}$ ) for the ${ }^{1} D_{2}$ partial wave for two choices of the cutoff: $\Lambda^{2}=4 m_{N}^{2}-\left(3 M_{\pi}\right)^{2}$ and $\Lambda^{2}=4 m_{N}^{2}-\left(4 M_{\pi}\right)^{2}$. A large difference between the two results indicates that one should have an additional condition to choose a physically correct solution. Currently we are trying to formulate such a condition in a mathematically correct way. 

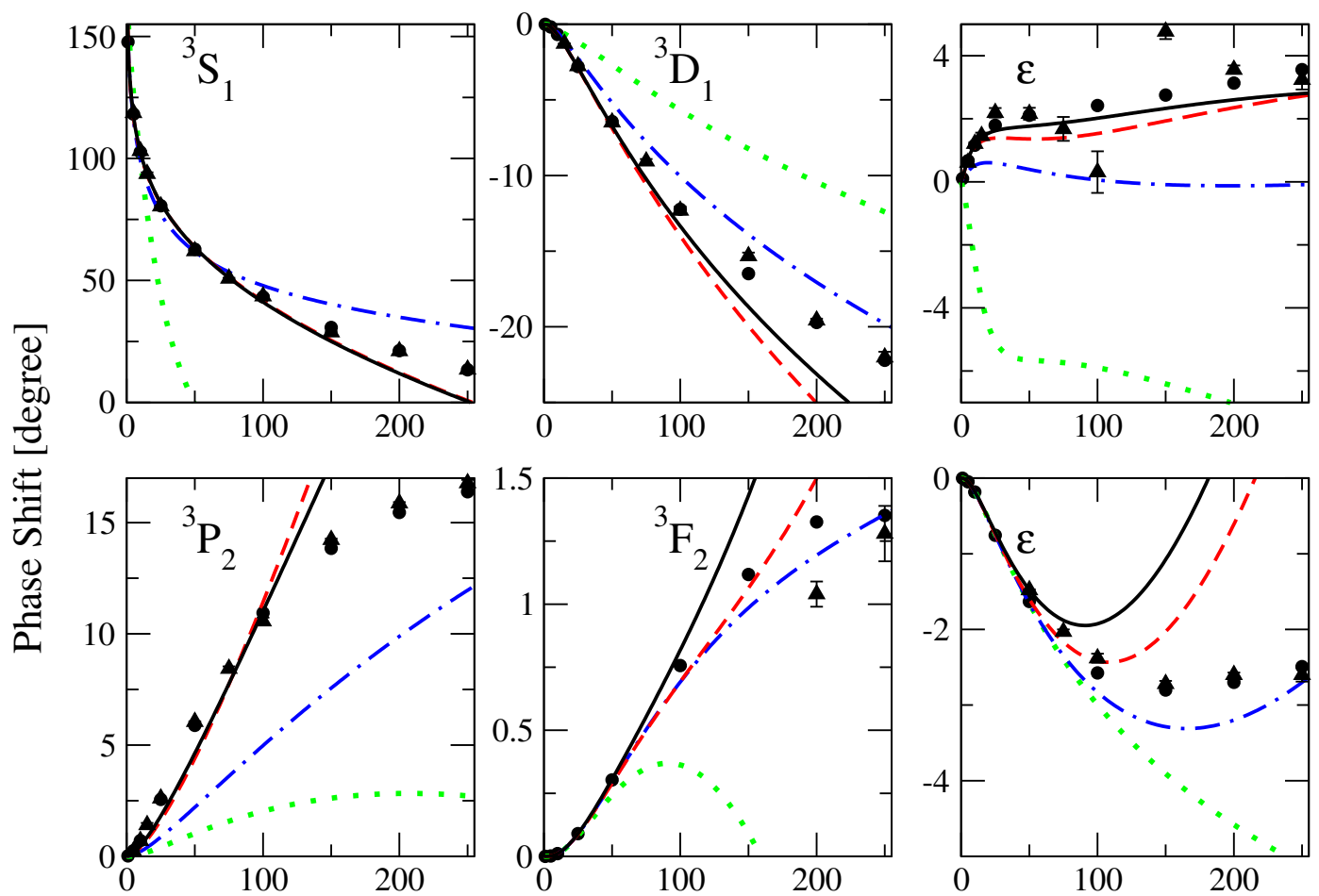

\section{Lab. Energy $[\mathrm{MeV}]$}

Figure 3: Neutron-proton phase shifts and mixing angles in the coupled ${ }^{3} S_{1}-{ }^{3} D_{1}$ and ${ }^{3} P_{2}-{ }^{3} F_{2}$ channels. For notation see Fig. 2.

\begin{tabular}{|l|c|c|c|}
\hline & ${ }^{3} S_{1}-{ }^{3} D_{1}, U_{11}$ & ${ }^{3} S_{1}-{ }^{3} D_{1}, U_{12}$ & ${ }^{3} P_{2}-{ }^{3} D_{2}, U_{11}$ \\
\hline$Q^{0}$ & $-2.95 \times 10^{2}$ & & \\
\hline$Q^{1}$ & $6.14 \times 10^{2}$ & & \\
\hline$Q^{2}$ & $2.08 \times 10^{2}$ & $-5.92 \times 10^{3}$ & $5.59 \times 10^{2}$ \\
\hline$Q^{3}$ & $2.07 \times 10^{2}$ & $-6.94 \times 10^{3}$ & $7.50 \times 10^{2}$ \\
\hline
\end{tabular}

Table 2: Matrix elements of the generalized potential at the matching point $U_{i j}\left(\mu_{M}^{2}\right)$ at different chiral orders after subtracting one-pion exchange contribution. Coupled partial waves.

\section{Summary}

The nucleon-nucleon interaction close to threshold is studied within an approach based on an analytic extrapolation of subthreshold amplitudes calculated in ChPT using partial-wave dispersion relations. The free parameters of the chiral Lagrangian are adjusted to the nucleon-nucleon phase shifts using Nijmegen partial-wave analysis. The obtained results are in a reasonable agreement with the empirical PWAs and are comparable with the ones obtained in the potential approach. A reasonable convergence for the subthreshold amplitude was demonstrated. We also address the question of the uniqueness of the obtained results. 


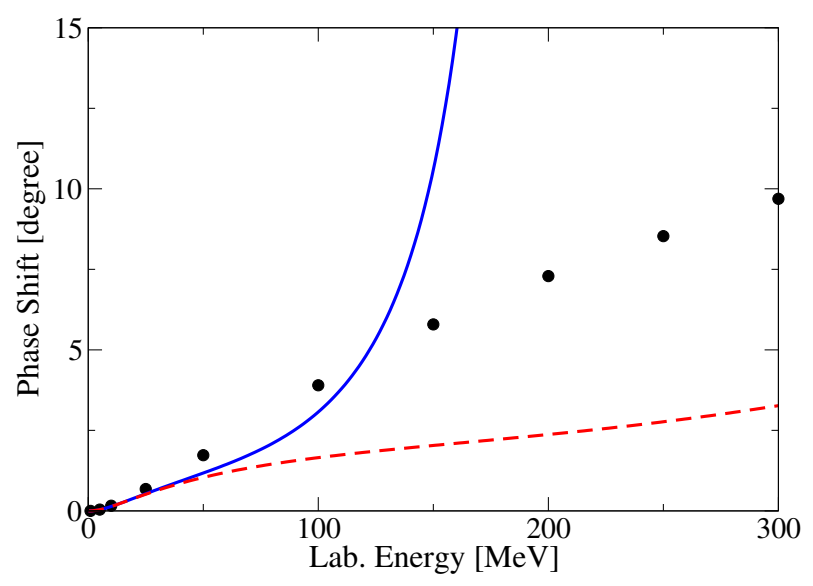

Figure 4: Predictions for the ${ }^{1} D_{2}$ partial wave. Solid line corresponds to $\Lambda^{2}=4 m_{N}^{2}-\left(3 M_{\pi}\right)^{2}$, dashed line corresponds to $\Lambda^{2}=4 m_{N}^{2}-\left(4 M_{\pi}\right)^{2}$. The data are from [21].

\section{Acknowledgment}

This work is supported by the Deutsche Forschungsgemeinschaft (SFB/TR 16, "Subnuclear Structure of Matter"), by the European Research Council (acronym NuclearEFT, ERC-2010-StG 259218).

\section{References}

[1] S. Scherer, Prog. Part. Nucl. Phys. 64, 1 (2010), 0908.3425

[2] S. Weinberg, Phys. Lett. B251, 288 (1990)

[3] N. Kaiser, R. Brockmann, W. Weise, Nucl.Phys. A625, 758 (1997), nucl-th/ 9706045

[4] R. Machleidt, D. Entem, Phys.Rept. 503, 1 (2011), 1105.2919

[5] E. Epelbaum, U.G. Meissner, Ann. Rev. Nucl. Part. Sci. 62, 159 (2012), 1201.2136

[6] D.B. Kaplan, M.J. Savage, M.B. Wise, Phys. Lett. B424, 390 (1998), nucl-th/ 9801034

[7] S. Fleming, T. Mehen, I.W. Stewart, Nucl. Phys. A677, 313 (2000), nucl-th / 9911001

[8] S. Weinberg, Physica A96, 327 (1979)

[9] A. Gasparyan, M.F.M. Lutz, Nucl.Phys. A848, 126 (2010), 1003.3426

[10] A. Gasparyan, M. Lutz, B. Pasquini, Nucl.Phys. A866, 79 (2011), 1102.3375

[11] I. Danilkin, M. Lutz, S. Leupold, C. Terschlusen, Eur.Phys.J. C73, 2358 (2013), 1211.1503

[12] A. Gasparyan, M. Lutz, E. Epelbaum, Eur.Phys.J. A49, 115 (2013), 1212 . 3057

[13] G.F. Chew, S. Mandelstam, Phys. Rev. 119, 467 (1960)

[14] M. Albaladejo, J. Oller, Phys.Rev. C84, 054009 (2011), 1107.3035

[15] M. Albaladejo, J. Oller, Phys.Rev. C86, 034005 (2012), 1201.0443

[16] Z.H. Guo, J. Oller, G. Rios (2013), 1305.5790 
[17] J.A. Oller (2014), 1402.2449

[18] I. Danilkin, A. Gasparyan, M. Lutz, Phys.Lett. B697, 147 (2011), 1009.5928

[19] M. Lutz, Nucl.Phys. A677, 241 (2000), nucl-th/9906028

[20] R. Higa, M. Robilotta, Phys.Rev. C68, 024004 (2003), nucl-th / 0304025

[21] V. Stoks, R. Klomp, M. Rentmeester, J. de Swart, Phys.Rev. C48, 792 (1993)

[22] N. A. Arndt, et al., SAID online program, http://gwdac.phys.gwu.edu (2013)

[23] R.A. Arndt, I.I. Strakovsky, R.L. Workman, Phys. Rev. C50, 2731 (1994), nucl-th/ 9407035

[24] L. Castillejo, R.H. Dalitz, F.J. Dyson, Phys. Rev. 101, 453 (1956)

[25] P.W. Johnson, R.L. Warnock, J. Math. Phys. 22, 385 (1981) 